\title{
Do patients with type 1 and type 2 diabetes really have an impaired endothelial function? A population-based propensity score matching analysis
}

Klaus Empen ${ }^{*}$, Roberto Lorbeer ${ }^{2}$, Henry Völzke ${ }^{2,5}$, Thorsten Reffelmann ${ }^{1,5}$, Sabine Schipf ${ }^{3}$, Matthias Nauck ${ }^{3,5}$, Wolfgang Kerner ${ }^{4}$, Henri Wallaschofski ${ }^{3,5}$, Stephan B Felix ${ }^{1,5}$ and Marcus Dörr ${ }^{1,5}$

\begin{abstract}
Background: Previous studies suggested an impaired endothelial function in patients with diabetes. However, the validity of this finding may be limited by the lack of adequate adjustment for further cardiovascular confounders. We assessed endothelial function as measured by flow-mediated dilation (FMD) of the brachial artery in patients with either type 1 or type 2 diabetes in comparison with non-diabetic controls.

Methods: The study population comprised 1487 subjects including 122 subjects with type 2 diabetes, aged 25 to 85, from the population-based Study of Health in Pomerania, and 65 outpatients, aged 23 to 75, with type 1 diabetes. FMD measurements were performed using standardized ultrasound techniques. Subjects with type 1 and type 2 diabetes were matched 1:4 to healthy controls using propensity score matching.

Results: Neither type 1 diabetes $(\beta=0.142$; $S E=0.568, p=0.803$ ) nor type 2 diabetes $(\beta=0.107 ; \mathrm{SE}=0.340$, $p=0.752$ ) were significantly associated with FMD in comparison with their non-diabetic controls after adjustment for major cardiovascular confounders like age, gender, body mass index, smoking status, hypertension, antihypertensive medication, LDL and HDL cholesterol levels.

Conclusions: In this population-based study comparing adjusted FMD values of diabetic individuals with adequately matched controls, propensity score analyses revealed no association between diabetes and endothelial function. Since these findings are in discordance with the majority of previous reports, we suggest performing similar analyses using data from other population-based studies.
\end{abstract}

Keywords: Type 1 diabetes, Type 2 diabetes, Flow-mediated dilation, FMD, Endothelial function, Epidemiology

\section{Background}

Today, the most extensively used non-invasive technique for assessment of endothelial function is sonographic determination of flow-mediated dilation (FMD) of the brachial artery [1]. FMD is considered to represent endothelium-dependent vasodilation [1]. Low FMD values predict cardiovascular events independently of established atherosclerosis [2]. Cardiovascular risk factors associated with endothelial dysfunction include hypertension [3],

\footnotetext{
* Correspondence: empen@uni-greifswald.de

'Department of Internal Medicine, Medizinische Klinik B, Universitätsmedizin, Greifswald, Sauerbruchstrasse, D-17475, Germany

Full list of author information is available at the end of the article
}

hypercholesterolemia [4], and smoking [5]. Furthermore, type 1 [6-11] and type 2 diabetes [12-17] were reported to be associated with endothelial dysfunction.

Previous studies suggesting an impaired endothelial function in patients with diabetes suffered partly from several limitations such as small sample sizes, different methods of FMD measurements or most importantly lack of adequate selection of control subjects [6-16] as well as non-adjustment for relevant cardiovascular confounders [18]. To overcome these limitations we performed the present propensity score matching analysis for comparison of FMD values of diabetic and non-diabetic 
subjects using population-based data of the Study of Health in Pomerania (SHIP).

\section{Methods}

SHIP was approved by the ethics committee of the University of Greifswald, and all participants gave informed written consent. Details on the design of SHIP have been published in detail previously [19].

\section{Controls}

Between March 2003 and October 2006, 1788 subjects volunteered for measurement of the FMD of the brachial artery. Exclusion criteria for the measurements were hypotension with systolic blood pressure $<100 \mathrm{~mm} \mathrm{Hg}$ $(n=15)$, the presence of any other medical contraindication $(n=4)$, and equipment malfunction $(n=36)$. The image quality of 215 FMD examinations was insufficient for appropriate readings. Exclusion of 122 subjects with a history of diabetes mellitus (type 2) according to physician's diagnosis or use of Antidiabetic medication as well as 31 subjects with hemoglobin A1c levels higher than the diagnostic cut off for diabetes of $\geq 6.5 \%$ resulted in a final non-diabetic control population of 1365 subjects (686 women).

\section{Cases}

The first case group consisted of 142 patients with type 1 diabetes recruited from a tertiary Diabetes Care Center in Karlsburg, West Pomerania. Data collection was performed between November 2005 and January 2007. Of these 142 patients, 68 subjects volunteered for measurement of the FMD of the brachial artery. Cases with insufficient image quality of FMD examinations $(n=3)$ were excluded. Consequently, this case group comprised 65 patients with type 1 diabetes (34 women). The second case group consisted of 122 subjects (54 women) with type 2 diabetes identified in the population-based SHIP.

\section{Measurements}

Information on socio-demographic characteristics, health behaviour, medication intake, and medical history was collected by computer-assisted personal interviews. In the present study we included age, gender, smoking and use of self-reported antihypertensive medication.

Somatometric measurements such as waist circumference, body mass index (BMI), blood pressure were measured as described in detail previously $[19,20]$. Hypertension was defined as a systolic and/or diastolic blood pressure of $\geq 140 \mathrm{mmHg}$ and $\geq 90 \mathrm{mmHg}$, respectively, or use of antihypertensive medication.

\section{Assays}

Non-fasting blood samples were drawn from the cubital vein in the supine position. The samples were taken between 07.00 a.m. and 04.00 p.m. and analyzed immediately for all parameters. Levels of low and high-density lipoprotein cholesterol (LDL-C and HDL-C), triglycerides and glucose, and HbA1c were determined according to the manufacturer's recommendations using standard assays as described in detail elsewhere [20]. Internal quality controls were performed at least daily.

\section{Endothelial function}

FMD was assessed as described in more detail previously [20-22] using standardized techniques. All measurements of brachial artery diameters were performed offline by independent sonographers and were subject to strict quality management [22]. In brief, FMD of the brachial artery was assessed by measuring the increase of the brachial artery diameter during reactive hyperemia after transient forearm ischemia induced by interrupting the blood flow for exactly 5 minutes via inflation of a blood pressure cuff placed around the forearm. Postischemia diameters were measured one minute after cuff release and restoration of forearm blood flow. Enddiastolic vessel diameters were measured from the anterior to the posterior M-line (i.e., the interface between the media and adventitia) of the vessel wall. Absolute FMD were calculated by subtracting baseline vessel diameters from post-ischemia vessel diameters. Relative changes were expressed as percentage of absolute FMD to baseline diameters.

\section{Statistical analyses}

Data on quantitative characteristics are expressed as median with minimum and maximum values. Data on qualitative characteristics are expressed as absolute numbers and percent values. Comparisons between diabetic and non-diabetic population were made using the Mann-Whitney U-test (continuous data) and $\chi^{2}$-test (nominal data). Multivariable linear regression models were performed calculating $\beta$-coefficients and standard error (SE) for change in FMD between diabetic (type 1 and 2) and non-diabetic subjects. The models were adjusted for age, gender, smoking, BMI, hypertension, antihypertensive medication, LDL cholesterol (LDL-C) and HDL cholesterol (HDL-C) levels.

We used propensity score matching to estimate FMD in subjects with similar characteristics of diabetic and non-diabetic groups. The likelihood of having type 1 or type 2 diabetes mellitus was modelled in a logistic regression depending on age, gender, BMI, current smoking, hypertension, LDL-C and HDL-C levels and the predicted probability was computed for each subject [23]. For propensity score matching we used the nearest neighbour matching method. In the event of ties, a control was selected at random from among all potential matches. 
For the power calculation we considered different ratios for propensity score matching (1:2, 1:3 or 1:4). The ratios were varied until a meaningful difference in FMD means of $1.5 \%$ was detectable with a power of $80 \%$ at least ( alpha $_{2 \text {-sided }}, 0.05$; common standard deviation, 3.8;). The ratio was increased until the power calculation resulted in a number of subjects with type 1 diabetes mellitus $\leq 65$. We chose a 1:4- matching where the power calculation resulted in a number of necessary subjects of 63, which was lower than the number patients with type 1 diabetes $(n=65)$. The final samples for the matched comparisons comprised 276 subjects for type 1 diabetes analyses (65 diabetic and 211 non-diabetic; power: $79.5 \%$ ) and 430 subjects for type 2 diabetes analyses (120 diabetic and 310 non-diabetic; power: 95.7\%) without missing data for confounder variables.

Bivariate comparisons were performed on the unmatched groups and multivariable regression analyses were performed on the matched groups. A value of $\mathrm{p}<0.05$ was considered statistically significant. All statistical analyses were performed using Stata 12.1 (Stata Corporation, College Station, TX, USA).

\section{Results}

Baseline characteristics of the non-diabetic and the diabetic groups are presented in Table 1. Subjects with type 1 diabetes showed a higher proportion of hypertension, increased HDL-C levels and decreased LDL-C levels as well as lower baseline diameter of the brachial artery compared to the non-diabetic group. There were no significant differences between these two groups concerning age, smoking status, BMI, systolic blood pressure, and FMD (Table 1). Subjects with type 2 diabetes were different from non-diabetic subjects in all analyzed parameters except the gender proportion.

Linear regression analyses revealed no difference in adjusted FMD between patients with type 1 diabetes and non-diabetic subjects $(\beta=0.142$; $\mathrm{SE}=0.568, \mathrm{p}=0.803$ ) (Table 2). Likewise, there was no difference in adjusted FMD between patients with diabetes type 2 and

Table 1 Characteristics of subjects with and without diabetes

\begin{tabular}{|c|c|c|c|}
\hline & $\begin{array}{l}\text { SHIP-population } \\
\text { Non diabetic } \\
n=1365\end{array}$ & $\begin{array}{c}\text { Type } 2 \text { diabetes } \\
\qquad n=122\end{array}$ & $\begin{array}{c}\text { Outpatients } \\
\text { Type } 1 \text { diabetes } \\
n=65\end{array}$ \\
\hline Age, yr & $51(25-85)$ & $63(31-84)^{\ddagger}$ & $50(23-75)$ \\
\hline Sex (male) & $679(49.7 \%)$ & $68(55.7 \%)$ & $31(47.7 \%)$ \\
\hline Smoking status & & $\neq$ & \\
\hline Never-smoker & $590(43.3 \%)$ & $48(39.3 \%)$ & $32(49.2 \%)$ \\
\hline Ex-smoker & $427(31.3 \%)$ & $61(50.0 \%)$ & $14(21.5 \%)$ \\
\hline Current smoker & $347(25.4 \%)$ & $13(10.7 \%)$ & $19(29.2 \%)$ \\
\hline Body mass index, $\mathrm{kg} / \mathrm{m}^{2}$ & $27.0(16.8-49.8)$ & $31.0(19.8-50.0)^{\ddagger}$ & $26.5(20.0-41.5)$ \\
\hline Waist circumference, $\mathrm{cm}$ & $91.3(57.3-155.0)$ & $103.0(75.2-135.0)^{\ddagger}$ & $91.2(63.0-126.1)$ \\
\hline Systolic blood pressure, $\mathrm{mmHg}$ & $129(89-219)$ & $135(90-208)^{\ddagger}$ & $137(101-179)$ \\
\hline Diastolic blood pressure, $\mathrm{mmHg}$ & $82(54-122)$ & $79(57-113)^{\dagger}$ & $78(47-101)^{*}$ \\
\hline Hypertension & $623(45.7 \%)$ & $92(75.4 \%)^{\ddagger}$ & $41(63.1)^{\dagger}$ \\
\hline Use of antihypertensive medication & $418(30.6 \%)$ & $98(80.3 \%)^{\ddagger}$ & $37(56.9 \%)^{\ddagger}$ \\
\hline HDL cholesterol, mmol// & $1.13(0.39-2.82)$ & $0.87(0.42-2.22)^{\ddagger}$ & $1.35(0.53-2.84)^{\ddagger}$ \\
\hline LDL cholesterol, mmol// & $3.46(0.73-11.03)$ & $3.19(1.45-5.99)^{*}$ & $2.98(1.64-5.06)^{\dagger}$ \\
\hline Triglycerides, mmol/l & $1.43(0.17-52.22)$ & $1.99(0.32-13.27)^{\ddagger}$ & $1.04(0.31-4.74)^{\ddagger}$ \\
\hline Use of sex hormones medication & $126(9.2 \%)$ & $1(0.8 \%)^{\dagger}$ & $2(3.1 \%)$ \\
\hline Glucose, mmol/l & $5.1(3.3-11.1)$ & $6.4(2.4-20.2)^{\ddagger}$ & $8.4(1.5-22.8)^{\ddagger}$ \\
\hline $\mathrm{HbA} 1 \mathrm{c}, \%$ & $5.2(2.8-6.4)$ & $6.5(3.7-10.1)^{\ddagger}$ & $7.5(4.3-11.7)^{\ddagger}$ \\
\hline Albumin, mg/l & $5.4(0-2120)$ & $10.6(0-2880)^{\ddagger}$ & $12.6(2.4-756)^{\ddagger}$ \\
\hline Baseline diameter A.brachialis, mm & $3.84(2.10-6.01)$ & $4.07(2.21-6.01)^{\ddagger}$ & $3.78(2.54-4.99)^{*}$ \\
\hline $\mathrm{FMD}, \%$ & $4.51(-8.44-29.41)$ & $3.89(-3.50-11.13)^{*}$ & $4.21(-1.32-14.79)$ \\
\hline
\end{tabular}

Data are given as number (percentage) or median (minimum-maximum).

${ }^{*} \mathrm{p}<0.05,{ }^{\dagger} \mathrm{p}<0.01,{ }^{\ddagger} \mathrm{p}<0.001 \mathrm{c} 2$-test (nominal data) or Mann-Whitney $U$ test (continuous data).

Comparisons were performed separately against non-diabetics.

$\mathrm{HDL}$, high-density-lipoprotein; LDL, low-density-lipoprotein; HbA1c, hemoglobin A1c; FMD, flow-mediated dilatation. 
Table 2 Multivariable linear regression analysis in volunteers with type 1 diabetes and non-diabetic matched controls with respect to FMD

\begin{tabular}{lccc}
\hline & \multicolumn{3}{c}{ FMD } \\
\cline { 2 - 4 } $\mathbf{N}=\mathbf{2 7 6}$ & $\boldsymbol{\beta}$-coefficients & SE & p-value \\
\hline Type 1 diabetes & 0.142 & 0.568 & 0.803 \\
Age, years & -0.067 & 0.023 & 0.004 \\
Sex (male) & -1.645 & 0.534 & 0.002 \\
Current smoking & -0.099 & 0.561 & 0.860 \\
Body mass index, $\mathrm{kg} / \mathrm{m}^{2}$ & 0.063 & 0.061 & 0.307 \\
Hypertension & -0.444 & 0.571 & 0.438 \\
Use of antihypertensive medication & -0.361 & 0.612 & 0.556 \\
HDL cholesterol, mmol// & 0.151 & 0.555 & 0.786 \\
LDL cholesterol, mmol// & 0.288 & 0.293 & 0.327 \\
\hline
\end{tabular}

FMD, flow-mediated dilatation; HDL, high-density-lipoprotein; LDL, low-density-lipoprotein.

non-diabetic subjects $(\beta=0.107 ; \mathrm{SE}=0.340, \mathrm{p}=0.752)$ (Table 3).

In a sensitivity analysis, there was no association between $\mathrm{HbA} 1 \mathrm{c}$ levels and FMD neither in type 1 diabetes $(\beta=-0.099 ; \mathrm{SE}=0.437, \mathrm{p}=0.821)$ nor in type 2 diabetes $(\beta=0.266$; $\mathrm{SE}=0.207, \mathrm{p}=0.201)$ adjusted for age, gender, smoking, BMI, hypertension, antihypertensive medication, LDL and HDL cholesterol levels. To minimize potential misclassification bias concerning undiagnosed diabetes in control subjects, we repeated propensity score matching with controls with an HbA1c level $<5.7 \%$. This analysis revealed similar results showing also no associations between diabetes and FMD neither for type 1 $(\beta=0.274$; $\mathrm{SE}=0.538, \mathrm{p}=0.611)$ nor for type 2 diabetes $(\beta=0.208$; SE $=0.338, \mathrm{p}=0.539)$.

Table 3 Multivariable linear regression analysis in volunteers with type 2 diabetes and non-diabetic matched controls with respect to FMD

\begin{tabular}{lccc}
\hline & \multicolumn{3}{c}{ FMD } \\
\cline { 2 - 4 } $\mathbf{N}=\mathbf{4 3 0}$ & $\boldsymbol{\beta}$-coefficients & SE & p-value \\
\hline Type 2 diabetes & 0.107 & 0.340 & 0.752 \\
Age, years & -0.072 & 0.014 & $<0.001$ \\
Sex, male & -1.418 & 0.328 & $<0.001$ \\
Current smoking & -0.040 & 0.450 & 0.930 \\
Body mass index, kg/m ${ }^{2}$ & -0.038 & 0.035 & 0.282 \\
Hypertension & 0.373 & 0.366 & 0.308 \\
Use of antihypertensive medication & -0.423 & 0.374 & 0.259 \\
HDL cholesterol, mmol/l & -0.118 & 0.467 & 0.801 \\
LDL cholesterol, mmol/l & -0.088 & 0.172 & 0.609 \\
\hline
\end{tabular}

FMD, flow-mediated dilatation; $\mathrm{HDL}$, high-density-lipoprotein; LDL, low-density-lipoprotein.

\section{Discussion}

In contrast to previous studies we did not find an association of either type 1 [6-11] or type 2 [12-16] diabetes with impaired endothelial function. Our deviating findings may be explained by a more sophisticated selection of controls by using propensity score matching and considering known confounders, i.e. gender, age, body mass index, smoking, hypertension, LDL-C and HDL-C levels, as well as use of antihypertensive medication.

Selection of healthy controls remains an issue of concern of many previous preclinical and clinical studies [24]. Controls in studies investigating the association between diabetes and endothelial function were often recruited from convenience samples like members of hospital staff, their relatives, or friends (e.g. [8]). However, lack of adequate control groups cannot be attributed to every single of previous studies investigating endothelial function in patients with type 1 diabetes. For example, cardiovascular risk factors appeared well-balanced between patients with type 1 diabetes and controls in one of these studies [25]. Interestingly, in this study FMD was lower in diabetic patients, but this association was no longer observed after correction for baseline diameters. However, participants of this study were significantly younger than in SHIP (median age 32 years) and consequently did not present further cardiovascular risk factors with the exception of smoking. According to a meta-analysis [26], the association of FMD with cardiovascular risk is limited to populations at relatively low cardiovascular risk. Due to the prevalence of further cardiovascular risk factors the resulting estimated general cardiovascular risk in our subjects was not as low as in other studies $[7,9]$. Thus, the relatively high cardiovascular risk of our volunteers might partly explain the lack of association of type 1 diabetes and FMD observed in the present study. In partial concordance with this assumption are the results of a recent study in children with type 1 diabetes with a disease duration $>5$ years who did not present with excessive additional cardiovascular risk revealing an impaired FMD in children with diabetes compared to controls [27]. In contrast, another recent study did not observe impaired FMD at baseline in children and adolescents with type 1 diabetes and a mean disease duration of 4 years, but did observe an impairment after a mean follow-up of 3 years [28]. Our volunteers were adults with a mean age of 50 years, mostly long-lasting type 1 diabetes, and a high prevalence of additional cardiovascular risk factors which makes a direct comparison difficult.

With respect to diabetes mellitus type 2 previous studies mostly described an association with decreased endothelial function. For example, the HOORN study with a larger sample size than ours described lower FMD values in type 2 diabetics compared to controls with normal 
glucose metabolism [13]. This association was still present after adjustment for conventional cardiovascular risk factors, which were of similar prevalence as in our group of subjects with type 2 diabetes. In this study, however, impaired glucose metabolism was not associated with impaired FMD. In a more recent study of 165 patients with diabetes mellitus type 2, FMD was inversely associated with duration of diabetes, but there was no association of FMD with other cardiovascular risk factors [17]. Unfortunately, we are unable to confirm this finding because precise data on disease duration are not available in a relevant proportion of our volunteers with type 2 diabetes.

Contradictory findings concerning the association of type 2 diabetes and FMD may be potentially partly explained by inconsistent definitions of type 2 diabetes. In our study, the definition was based on self-reported physician's diagnosis or use of antidiabetic medication. Although volunteers with an HbA1c value greater than $6.5 \%$ were excluded from the control group, because they can be considered diabetic according to current diagnostic guidelines [29], we cannot completely exclude that an uncertain proportion of control volunteers had undiagnosed type 2 diabetes leading to a misclassification bias. However, the probability of including a relevant proportion of subjects with undiagnosed type 2 diabetes in the controls appears low since reanalysis of the data after including only subjects with HbA1c levels lower than $5.7 \%$ as controls did not reveal different results.

In our sensitivity analysis, there were no associations of HbA1c levels with FMD, neither in type 1 nor in type 2 diabetes. In a previous study in a non-diabetic population, we observed an inverse association of HbA1c only in women, but not in men [20]. In the current study, we considered numbers of subjects with diabetes too low for performing gender-specific analyses. Most previous authors reported impaired endothelial function in type 1 and 2 diabetes, but not all investigators did confirm this association [25]. Considering the effects of publication bias, the association may be truly neutral - as suggested by our data analysis.

Major strengths of our study are the population-based design, the comprehensive and detailed assessment of potential metabolic and cardiovascular confounding factors [22], the size of the study population, the accurate FMD measurement under strict quality management by standardised protocol and certified staff [22], and the use of forearm ischemia-induced FMD [1]. Forearm ischemia-induced FMD precludes the potential contribution of ischemia of the brachial artery itself - in contrast to upper arm ischemia-induced FMD which is known to induce greater vasodilation [1]. Finally, and probably most importantly, our analysis is the first one selecting controls out of the same population by propensity score matching.

\section{Conclusion}

In the present analysis, there was no association of either type 1 or type 2 diabetes with impaired endothelial function as measured by FMD. Since these findings are in discordance with the majority of previous reports, we suggest performing similar analyses using data from other population-based studies.

\section{Abbreviations}

BMI: Body mass index; FMD: Flow-mediated dilation; HDL-C: High density lipoprotein cholesterol; LDL-C: Low density lipoprotein cholesterol;

SE: Standard error; SHIP: Study of Health in Pomerania.

\section{Competing interests}

The authors declare that they have no competing interests.

\section{Authors' contributions}

$K E, R L, M D$ designed the study, researched data, and wrote the first draft of the manuscript. RL performed statistical analyses. HV, TR, SS, MN, HW, SBF researched data, contributed to discussion, and reviewed the manuscript. WK recruited patients with type 1 diabetes and reviewed the manuscript. All authors have approved the final version of the manuscript.

\section{Acknowledgements}

This work was supported by grants from the German Federal Ministry of Education and Research (BMBF, Grants 01ZZ0403, 01ZZ0103, 01GI0883), the Ministry for Education, Research and Cultural Affairs as well as the Ministry of Social Affairs of the Federal State of Mecklenburg-West Pomerania. Furthermore, this work is part of the research project Greifswald Approach to Individualised Medicine (GANI_MED). The GANI_MED consortium is funded by the German Federal Ministry of Education and Research and as well as by the Ministry of Cultural Affairs of the German Federal State of Mecklenburg West Pomerania (03IS2061A). This study was further supported by the DZHK (German Centre for Cardiovascular Research).

The contributions to data collection made by field workers, study physicians, ultrasound technicians, interviewers, and computer assistants are gratefully acknowledged.

\section{Author details}

${ }^{1}$ Department of Internal Medicine, Medizinische Klinik B, Universitätsmedizin, Greifswald, Sauerbruchstrasse, D-17475, Germany. ${ }^{2}$ Institute for Community Medicine, University Medicine, Greifswald 17475, Germany. ${ }^{3}$ Institute for Clinical Chemistry and Laboratory Medicine, University Medicine, Greifswald 17475, Germany. ${ }^{4}$ Diabetes Center, Karlsburg 17495, Germany. ${ }^{5}$ DZHK (German Center for Cardiovascular Research), partner site Greifswald, Greifswald 17475, Germany.

Received: 14 October 2013 Accepted: 2 December 2013 Published: 5 December 2013

\section{References}

1. Corretti MC, Anderson TJ, Benjamin EJ, Celermajer D, Charbonneau F, Creager MA, Deanfield J, Drexler H, Gerhard-Herman M, Herrington D, et al: Guidelines for the ultrasound assessment of endothelial-dependent flow-mediated vasodilation of the brachial artery: a report of the international brachial artery reactivity task force. J Am Coll Cardiol 2002, 39(2):257-265.

2. Yeboah J, Folsom AR, Burke GL, Johnson C, Polak JF, Post W, Lima JA, Crouse JR, Herrington DM: Predictive value of brachial flow-mediated dilation for incident cardiovascular events in a population-based study: the multi-ethnic study of atherosclerosis. Circulation 2009, 120(6):502-509.

3. Benjamin EJ, Larson MG, Keyes MJ, Mitchell GF, Vasan RS, Keaney JF Jr, Lehman BT, Fan S, Osypiuk E, Vita JA: Clinical correlates and heritability of flow-mediated dilation in the community: the Framingham heart study. Circulation 2004, 109(5):613-619. 
4. Simons LA, Sullivan D, Simons J, Celermajer DS: Effects of atorvastatin monotherapy and simvastatin plus cholestyramine on arterial endothelial function in patients with severe primary hypercholesterolaemia. Atherosclerosis 1998, 137(1):197-203.

5. Celermajer DS, Sorensen KE, Georgakopoulos D, Bull C, Thomas O, Robinson J, Deanfield JE: Cigarette smoking is associated with dose-related and potentially reversible impairment of endothelium-dependent dilation in healthy young adults. Circulation 1993, 88(5 Pt 1):2149-2155.

6. Ce GV, Rohde LE, Da Silva AM, Punales MK, De Castro AC, Bertoluci MC: Endothelial dysfunction is related to poor glycemic control in adolescents with type 1 diabetes under 5 years of disease: evidence of metabolic memory. J Clin Endocrinol Metab 2011, 96(5):1499-1493.

7. Chan NN, Vallance P, Colhoun HM: Endothelium-dependent and -independent vascular dysfunction in type 1 diabetes: role of conventional risk factors, sex, and glycemic control. Arterioscler Thromb Vasc Biol 2003, 23(6):1048-1054

8. Clarkson P, Celermajer DS, Donald AE, Sampson M, Sorensen KE, Adams M, Yue DK, Betteridge DJ, Deanfield JE: Impaired vascular reactivity in insulindependent diabetes mellitus is related to disease duration and low density lipoprotein cholesterol levels. J Am Coll Cardiol 1996, 28(3):573-579.

9. Johnstone MT, Creager SJ, Scales KM, Cusco JA, Lee BK, Creager MA: Impaired endothelium-dependent vasodilation in patients with insulindependent diabetes mellitus. Circulation 1993, 88(6):2510-2516.

10. Sibal L, Agarwal SC, Schwedhelm E, Luneburg N, Boger RH, Home PD: A study of endothelial function and circulating asymmetric dimethylarginine levels in people with Type 1 diabetes without macrovascular disease or microalbuminuria. Cardiovasc Diabetol 2009, $8: 27$.

11. Thorne S, Mullen MJ, Clarkson P, Donald AE, Deanfield JE: Early endothelial dysfunction in adults at risk from atherosclerosis: different responses to L-arginine. J Am Coll Cardiol 1998, 32(1):110-116.

12. Donald AE, Halcox JP, Charakida M, Storry C, Wallace SM, Cole TJ, Friberg P, Deanfield JE: Methodological approaches to optimize reproducibility and power in clinical studies of flow-mediated dilation. J Am Coll Cardiol 2008, 51(20):1959-1964.

13. Henry RM, Ferreira I, Kostense PJ, Dekker JM, Nijpels G, Heine RJ, Kamp O, Bouter LM, Stehouwer CD: Type 2 diabetes is associated with impaired endothelium-dependent, flow-mediated dilation, but impaired glucose metabolism is not; the hoorn study. Atherosclerosis 2004, 174(1):49-56.

14. Irace C, Tschakovsky ME, Carallo C, Cortese C, Gnasso A: Endothelial dysfunction or dysfunctions? Identification of three different FMD responses in males with type 2 diabetes. Atherosclerosis 2008, 200(2):439-445.

15. Reyes-Soffer G, Holleran S, Di Tullio MR, Homma S, Boden-Albala B, Ramakrishnan R, Elkind MS, Sacco RL, Ginsberg HN: Endothelial function in individuals with coronary artery disease with and without type 2 diabetes mellitus. Metabolism 2010, 59(9):1365-1371.

16. Williams SB, Cusco JA, Roddy MA, Johnstone MT, Creager MA: Impaired nitric oxide-mediated vasodilation in patients with non-insulindependent diabetes mellitus. J Am Coll Cardiol 1996, 27(3):567-574.

17. Naka KK, Papathanassiou K, Bechlioulis A, Kazakos N, Pappas K, Tigas S, Makriyiannis D, Tsatsoulis A, Michalis LK: Determinants of vascular function in patients with type 2 diabetes. Cardiovasc Diabetol 2012, 11:127.

18. von Elm E, Altman DG, Egger M, Pocock SJ, Gotzsche PC, Vandenbroucke JP: The Strengthening the Reporting of Observational Studies in Epidemiology (STROBE) statement: guidelines for reporting observational studies. Ann Intern Med 2007, 147(8):573-577.

19. Volzke H, Alte D, Schmidt CO, Radke D, Lorbeer R, Friedrich N, Aumann N, Lau K, Piontek M, Born G, et al: Cohort profile: the study of health in Pomerania. Int J Epidemiol 2011, 40(2):294-307.

20. Lorbeer R, Empen K, Dorr M, Arndt M, Schipf S, Nauck M, Wallaschofski H, Felix SB, Volzke H: Association between glycosylated haemoglobin A(1c) and endothelial function in an adult non-diabetic population. Atherosclerosis, 217(2):358-363.

21. Empen K, Lorbeer R, Dorr M, Haring R, Nauck M, Glaser S, Krebs A Reffelmann $T$, Ewert R, Volzke $H$, et al: Association of testosterone levels with endothelial function in Men: results from a population-based study. Arterioscler Thromb Vasc Biol 2012, 32:481-486.

22. Volzke $H$, Robinson DM, Spielhagen $T$, Nauck M, Obst A, Ewert R, Wolff $B$, Wallaschofski H, Felix SB, Dorr M: Are serum thyrotropin levels within the reference range associated with endothelial function? Eur Heart J 2009, 30(2):217-224

23. Leuven ESB: PSMATCH2: Stata module to perform full Mahalanobis and propensity score matching, common support graphing, and covariate imbalance testing; 2003.

24. Feinstein AR: Experimental requirements and scientific principles in case-control studies. J Chronic Dis 1985, 38(2):127-133.

25. Lambert J, Aarsen M, Donker AJ, Stehouwer CD: Endothelium-dependent and -independent vasodilation of large arteries in normoalbuminuric insulin-dependent diabetes mellitus. Arterioscler Thromb Vasc Biol 1996, 16(5):705-711.

26. Witte DR, Westerink J, de Koning EJ, van der Graaf Y, Grobbee DE, Bots ML: Is the association between flow-mediated dilation and cardiovascular risk limited to low-risk populations? J Am Coll Cardiol 2005, 45(12):1987-1993.

27. Ciftel M, Ertug H, Parlak M, Akcurin G, Kardelen F: Investigation of endothelial dysfunction and arterial stiffness in children with type 1 diabetes mellitus and the association with diastolic dysfunction. Diab Vasc Dis Res 2013. Epub ahead of print] doi:10.1177/1479164113508564.

28. Bruzzi P, Predieri B, Patianna VD, Salvini A, Rossi R, Modena MG, lughetti L: Longitudinal evaluation of Endothelial Function in children and adolescents with Type 1 Diabetes Mellitus: a long term follow up study. Pediatr Int 2013. doi:10.1111/ped.12220. [Epub ahead of print.

29. Diagnosis and classification of diabetes mellitus. Diabetes Care 2010, 33(Suppl 1):S62-S69.

\section{doi:10.1186/1475-2840-12-174}

Cite this article as: Empen et al:: Do patients with type 1 and type 2 diabetes really have an impaired endothelial function? A populationbased propensity score matching analysis. Cardiovascular Diabetology 2013 12:174.

\section{Submit your next manuscript to BioMed Central and take full advantage of:}

- Convenient online submission

- Thorough peer review

- No space constraints or color figure charges

- Immediate publication on acceptance

- Inclusion in PubMed, CAS, Scopus and Google Scholar

- Research which is freely available for redistribution 